\title{
Biological Evaluation of Phellinsin A Analogues as Chitin Synthase II Inhibitors
}

\author{
Sangku Lee." Sung-Uk Kim, Eungsoo Kim. Eui-Il Hwang, Sang-Hun Jung, and Hyeong Kyu Lee \\ Natural Medicine Research Center, KRIBB, Daejeon 305-806, Korea. "E-mail. hykylee@kribb,re.kr.

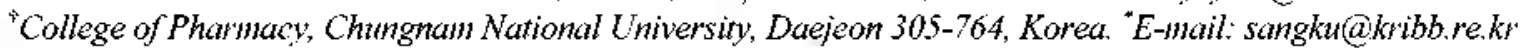 \\ Received July 5, 2007
}

Key Words : Inhibition, $\gamma$-Lactone, Antifungal agents

Chitin is the $\beta$-1,4-linked homopolymer of $N$-acetyl-Dglucosamine (GlcNAc) that constitutes an important structural component of the cell walls of nearly all zoopathogenic and phytopathogenic fungi, and plays a crucial role in the determination of cell morphology.' Chitin have been known to be synthesized by chitin synthase I, II, and III in Saccharomyces cerevisiae." Chitin synthase II is an essential enzyme for the formation of primary septum between mother and daughter cells, ${ }^{3}$ and chitin synthase III is responsible for the formation of chitin ring at bud emergence, ${ }^{4}$ whereas chitin synthase I is a repair enzyme of the damaged cells on cell division." Accordingly, specific inhibitors of chitin synthase II and III are expected to be an attractive target for the development of antifungal agents.

In the course of exploring a chitin synthase II inhibitor from microbial sources, we isolated a new phenolic lignan bearing a $\gamma$-lactone ring from the cultured broth of Phellinus sp. PL3. The lignan compound named phellinsin A (3a) exhibited inhibition of chitin synthase II with an $\mathrm{IC}_{50}$ value of $27 \mu \mathrm{g} / \mathrm{mL}$ and showed 2.5 times stronger inhibitory activity than polyoxin $\mathrm{D}$, and its structure was established by NMR analysis and synthesis. ${ }^{7}$ Our efforts toward the development of a potent antifungal agent have focused on examining structure-activity relationships for phellinsin A. We have modified the aryl group in phellinsin A by changing the number of phenolic $\mathrm{OH}$ groups in order to investigate the effect of the number of free phenolic $\mathrm{OH}$ groups in $\gamma$-lactone analogues of phellinsin A (3a) on chitin synthase II inhibitory activity. Herein, we describe the biological evaluation of phellinsin A analogues 3 .
The $\gamma$-lactone analogues 3c-1 of phellinsin A were prepared by oxidative dimerization of cinnamic acid derivatives 1 into the corresponding dilactones 2 , followed by monohydrolysis of the dilactones as shown in Scheme $1{ }^{7.8}$ The $\gamma$ lactones $\mathbf{3} \mathbf{a}$ and $\mathbf{3} \mathbf{b}$ were prepared by demethylation of compounds $\mathbf{3 d}$ and $\mathbf{3 e}$ using $\mathrm{BBr}_{3}$, respectively.

The $\gamma$-lactone analogues 3 of phellinsin A were examined for structure-activity relationships of phellinsin A (3a). Inhibitory activities of the compounds against chitin synthase II were evaluated by measurement of the formation of chitin with UDP-[ $\left.{ }^{14} \mathrm{C}\right]-\mathrm{N}$-acetyl-D-glucosamine. Table 1 showed the inhibitory activities of phellinsin $\mathrm{A}$ analogues at 140 and $280 \mu \mathrm{g} / \mathrm{mL}$ concentrations. The activities were largely dependent on the presence of free phenolic $\mathrm{OH}$ substituents in $\gamma$-actone analogues 3 . Generally, the compound 3f with non-phenolic $\mathrm{OH}$ groups did not show

Table 1. Inhibitory activities of phellinsin $\Lambda$ analog against chitin synthase II

\begin{tabular}{ccc}
\hline compound & $\begin{array}{r}\text { \% Inhibition } \\
(140 \mu \mathrm{g} / \mathrm{mL})\end{array}$ & $\begin{array}{c}\text { \% Inhibition } \\
(280 \mu \mathrm{g} / \mathrm{mL})^{b}\end{array}$ \\
\hline 3a & 83.2 & $-c$ \\
3b & 87.8 & $-c$ \\
3c & $\mathrm{NA}^{d}$ & 36.0 \\
3d & 3.6 & 40.1 \\
3e & 10.1 & 44.4 \\
3f & $\mathrm{NA}^{d}$ & 10.1 \\
\hline
\end{tabular}

"Percent inhibition at $140 \mu \mathrm{g} / \mathrm{mL}$. 'Percent inhibition at $280 \mu \mathrm{g} / \mathrm{mL}$. "Not detemined. NA, no inhibitory activity.

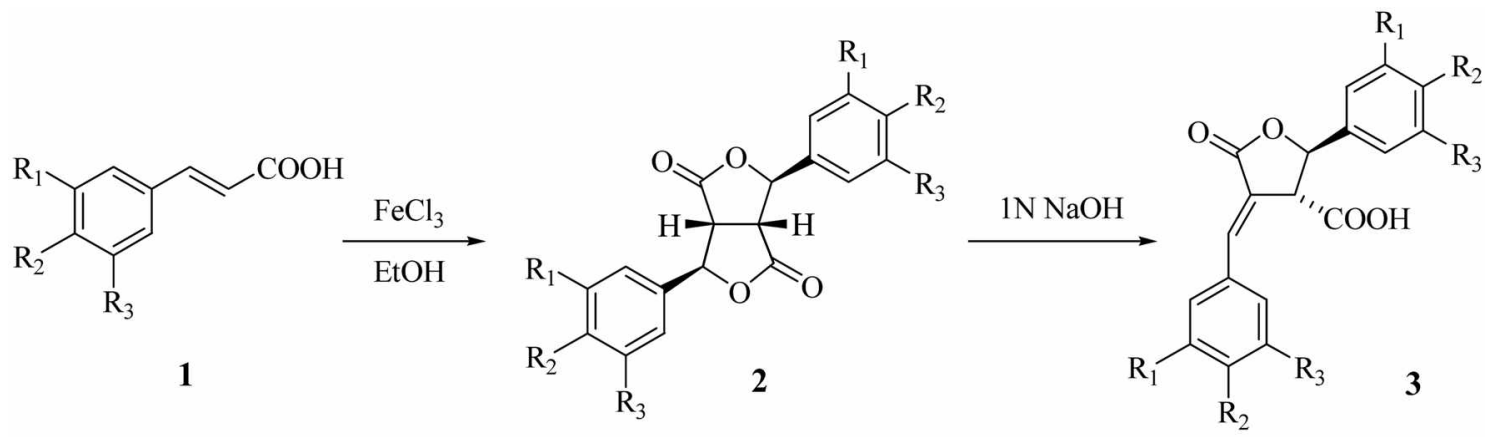

a. $\mathrm{R}_{1}=\mathrm{H}, \mathrm{R}_{2}=\mathrm{OH}, \mathrm{R}_{3}=\mathrm{OH}$; b. $\mathrm{R}_{1}=\mathrm{OH}, \mathrm{R}_{2}=\mathrm{OH}, \mathrm{R}_{3}=\mathrm{OH}$; c. $\mathrm{R}_{1}=\mathrm{H}, \mathrm{R}_{2}=\mathrm{OH}, \mathrm{R}_{3}=\mathrm{H}$;

d. $\mathrm{R}_{1}=\mathrm{H}, \mathrm{R}_{2}=\mathrm{OH}, \mathrm{R}_{3}=\mathrm{OCH}_{3}$; e. $\mathrm{R}_{1}=\mathrm{OCH}_{3}, \mathrm{R}_{2}=\mathrm{OH}, \mathrm{R}_{3}=\mathrm{OCH}_{3}$; f. $\mathrm{R}_{1}=\mathrm{H}, \mathrm{R}_{2}=\mathrm{H}, \mathrm{R}_{3}=\mathrm{H}$ 
Table 2. $\mathrm{IC}_{50}$ values of selected compounds

\begin{tabular}{cc}
\hline compound & $\mathrm{IC}_{50}(\mu \mathrm{g} / \mathrm{mL})$ \\
\hline 3a & 27.0 \\
3b & 9.6 \\
PD $^{\prime \prime}$ & 70.0 \\
\hline
\end{tabular}

'Polyoxin D as a reference.

inhibitory activities at $140 \mathrm{\mu g} / \mathrm{mL}$ concentration. Compounds 3a-b with two and three phenolic $\mathrm{OH}$ groups exhibited potent activities, whereas compound $3 \mathrm{c}$ with one phenolic $\mathrm{OH}$ group did not show inhibitory activities at $140 \mathrm{~kg} / \mathrm{mL}$ concentration. However, compounds 3d-e with one phenolic $\mathrm{OH}$ group and additional methoxy groups showed mild inhibitory activities at 140 and $280 \mathrm{mg} / \mathrm{mL}$ concentration, and $3 \mathrm{e}$ with two additional methoxy groups showed stronger activity than 3d with one additional methoxy group. Compound $3 \mathbf{b}$ possessing three phenolic $\mathrm{OH}$ groups showed most potent activity. Compounds $\mathbf{3 a}$ and $\mathbf{3 b}$, which showed potent inhibitory activities at $140 \mu \mathrm{g} / \mathrm{mL}$ concentration, were further evaluated with polyoxin $\mathrm{D}$ as positive control (Table 2). As shown in Table 2 , the $\gamma$-lactone analogue $\mathbf{3 b}\left(\mathrm{IC}_{50}=\right.$ $9.6 \mu \mathrm{g} / \mathrm{mL}$ ) possessing three phenolic $\mathrm{OH}$ groups at each aryl moiety exhibited about 3 times stronger inhibitory activity than phellinsin $\mathrm{A}(3 \mathrm{a})\left(\mathrm{IC}_{50}=27.0 \mu \mathrm{g} / \mathrm{mL}\right)$ possessing two phenolic $\mathrm{OH}$ groups. Compound $\mathbf{3 b}$ showed about 7 times more potent activity than polyoxin $\mathrm{D}\left(\mathrm{IC}_{50}=70.0 \mathrm{~kg} /\right.$ $\mathrm{mL}$ ). Structure-activity analysis indicated that the number of free phenolic $\mathrm{OH}$ groups in each aryl group of phellinsin $\mathrm{A}$ analogues contributed to increase the activity.

In conclusion, the effects of the number of free phenolic $\mathrm{OH}$ groups in phellinsin $\mathrm{A}$ analogues on inhibitory activities of chitin synthase II were examined for structure-activity relationships. Phellinsin A analogues lacking free phenolic $\mathrm{OH}$ functionality exhibited poor inhibitory activities. Compounds possessing three free phenolic $\mathrm{OH}$ groups in each aryl group of $\gamma$-lactones 3 showed more potent activity than those of one $\mathrm{OH}$ group or two $\mathrm{OH}$ groups. Compound $3 \mathrm{~b}$ exhibited about 3 times stronger inhibitory activity than phellinsin A (3a) and about 7 times more potent activity than polyoxin D.

\section{Experimental Section}

Typical procedure for preparation of compound $3 \mathrm{~b}$. To a solution of iron(III) chloride $(6.4 \mathrm{~g}, 28.5 \mathrm{mmol})$ in ethanol $(70 \mathrm{~mL})$ was added a solution of 3,5-dimethoxy-4-hydroxycinnamic acid $(1 \mathrm{~b}, 4.0 \mathrm{~g}, 17.8 \mathrm{mmol})$ in ethanol $(20 \mathrm{~mL})$ at room temperature. The reaction mixture was stirred for $1 \mathrm{~h}$ and concentrated in vacto. The residue was diluted with water, extracted with ethyl acetate, washed with brine, dried over $\mathrm{MgSO}_{4}$, and concentrated in vacto. The resulting residue was chromatographed on silica gel (1:1 hexaneEtOAc) to afford $4.9 \mathrm{~g}(61 \%)$ of dilactone $2 \mathrm{~b}$ as a pale brown solid: ${ }^{1} \mathrm{H} N \mathrm{MR}\left(300 \mathrm{MHz}\right.$, acetone- $\left.d_{6}\right) \delta 7.47(\mathrm{~s}, 2 \mathrm{H})$, 6.75 (s, 4H), 5.76 (brs, 2H), 4.12 (brs, $2 \mathrm{H}), 3.84$ (s, 12H); ${ }^{13} \mathrm{C}$ NMR $\left(75 \mathrm{MHz}\right.$, acetone- $\left.d_{6}\right) \delta 176.7,149.7,138.2$,
$130.5,105.0,84.1,57.4,49.8$; HRMS (FAB) $m / z 447.1288$ $\left[(\mathrm{M}+\mathrm{H})^{+}\right.$, calcd for $\left.\mathrm{C}_{22} \mathrm{H}_{23} \mathrm{O}_{10} 447.1291\right]$. To a solution of dilactone $2 \mathrm{~b}(1.0 \mathrm{~g}, 2.23 \mathrm{mmol})$ in THF $(10 \mathrm{~mL})$ was added $1 \mathrm{~N} \mathrm{NaOH}(50 \mathrm{~mL})$ at room temperature. The reaction mixture was stirred for $5 \mathrm{~min}$, acidified with $2 \mathrm{~N} \mathrm{HCl}$ to $\mathrm{pH}$ 4 , extracted with ethyl acetate, dried over $\mathrm{MgSO}_{4}$, and concentrated in vacuo. The resulting residue was purified by flash chromatography on silica gel $\left(9: 1 \mathrm{CH}_{2} \mathrm{Cl}_{2}-\mathrm{CH}_{3} \mathrm{OH}\right)$ to afford $0.6 \mathrm{~g}(60 \%)$ of $3 \mathrm{e}$ as a yellow solid: ${ }^{1} \mathrm{H}$ NMR $(300$ $\left.\mathrm{MHz}_{3} \mathrm{CDCl}_{3}\right) \delta 7.69(\mathrm{~d}, J=1.8 \mathrm{~Hz}, 1 \mathrm{H}), 6.77(\mathrm{~s}, 2 \mathrm{H}), 6.5 \mathrm{l}$ $(\mathrm{s}, 2 \mathrm{H}), 5.69(\mathrm{~d}, J=2.4 \mathrm{~Hz}, 1 \mathrm{H}), 4.12(\mathrm{~m}, 1 \mathrm{H}), 3.85(\mathrm{~s}, 6 \mathrm{H})$, $3.84(\mathrm{~s}, 6 \mathrm{H}) ;{ }^{13} \mathrm{C}$ NMR $\left(75 \mathrm{MHz}, \mathrm{CDCl}_{3}\right) \delta 174.5,171.4$, $147.4,147.2,141.9,137.7,135.2,130.2,124.4,118.1$, $107.7,101.9,80.5,56.4,56.3,53.5$; HRMS (FAB) $\mathrm{m} / \mathrm{z}$ 447.1286 [(M+H) ${ }^{+}$, calcd for $\mathrm{C}_{22} \mathrm{H}_{23} \mathrm{O}_{10}$ 447.1291]. To solution of compound $3 e(100 \mathrm{mg}, 0.22 \mathrm{mmol})$ in methylene chloride $(8 \mathrm{~mL})$ at $0^{\circ} \mathrm{C}$ was added $1 \mathrm{M} \mathrm{BBr}_{3}$ in $\mathrm{CH}_{2} \mathrm{Cl}_{2}(5$ $\mathrm{mL}, 5.0 \mathrm{mmol})$. The mixture was stirred for $2 \mathrm{~h}$ at $0^{\circ} \mathrm{C}$, quenched with $0.1 \mathrm{~N} \mathrm{HCl}$, extracted with ethyl acetate, dried over $\mathrm{MgSO}_{4}$, and concentrated in vacuo. The resulting residue was purified by a YMC ODS-H80 column chromatography $\left(2: 3 \mathrm{H}_{2} \mathrm{O}-\mathrm{CH}_{3} \mathrm{OH}\right)$ to afford $20 \mathrm{mg}(22 \%)$ of $3 \mathrm{~b}$ as a brown solid: 'H NMR (300 MHz, $\left.\mathrm{CD}_{3} \mathrm{OD}\right) \delta 7.41$ (d, $J=1.8$ $\mathrm{Hz}, 1 \mathrm{H}), 6.69(\mathrm{~s}, 2 \mathrm{H}), 6.30(\mathrm{~s}, 2 \mathrm{H}), 5.50(\mathrm{~d}, J=2.4 \mathrm{~Hz}, 1 \mathrm{H})$, $3.98(\mathrm{~m}, 1 \mathrm{H}) ;{ }^{13} \mathrm{C}$ NMR $\left(75 \mathrm{MHz}, \mathrm{CD}_{3} \mathrm{OD}\right) \delta 177.7174 .9$, $147.3,147.0,137.7,132.9,126.3,122.1,111.3,105.5,84.0$, 57.5; HRMS (FAB) $m / z \quad 391.0678\left[(\mathrm{M}+\mathrm{H})^{+}\right.$, calcd for $\left.\mathrm{C}_{18} \mathrm{H}_{15} \mathrm{O}_{10} 391.0665\right]$.

Compound 3a. Compound $\mathbf{3 a}$ was prepared from 4hydroxy-3-methoxycinnamic acid according to the typical procedure: ${ }^{1} \mathrm{H}$ NMR $\left(300 \mathrm{MHz}, \mathrm{CD}_{3} \mathrm{OD}\right) \delta 7.48(\mathrm{~s}, 1 \mathrm{H})$, $7.17(\mathrm{~d}, J=0.6 \mathrm{~Hz}, 1 \mathrm{H}), 7.05$ (d, $J=5.1 \mathrm{~Hz}, 1 \mathrm{H}), 6.79$ (d, $J$ $=4.8 \mathrm{~Hz}, 1 \mathrm{H}), 6.73(\mathrm{~d}, J=5.1 \mathrm{~Hz}, 1 \mathrm{H}), 6.71(\mathrm{~d}, J=0.9 \mathrm{~Hz}$, $1 \mathrm{H}$ ), 6.64 (dd, $J=4.8,0.9 \mathrm{~Hz}, 1 \mathrm{H}), 5.59$ (d, $J=0.9 \mathrm{~Hz}, 1 \mathrm{H}$ ), $3.97(\mathrm{~s}, \mathrm{HH}) ;{ }^{13} \mathrm{C} \mathrm{NMR}\left(75 \mathrm{MHz}, \mathrm{CD}_{3} \mathrm{OD}\right) \delta 178.2,175.2$, $149.4,146.8,146.7,146.5,140.3,133.6,127.4,125.5$, $122.6,118.3,118.0,116.6,116.4,113.4,84.3,57.7$; HRMS (FAB) $m / z 359.0771\left[(\mathrm{M}+\mathrm{H})^{+}\right.$, calcd for $\left.\mathrm{C}_{18} \mathrm{H}_{15} \mathrm{O}_{8} 359.0767\right]$.

Compound 3c: 'H NMR (300 MHz, CD $30 D) \delta 7.57(\mathrm{~s}$, $1 \mathrm{H}), 7.56$ (d, $J=8.7 \mathrm{~Hz}, 2 \mathrm{H}), 7.12$ (d, $J=9.0 \mathrm{~Hz}, 2 \mathrm{H}), 6.81$ $(\mathrm{d}, J=9.0 \mathrm{~Hz}, 2 \mathrm{H}), 6.76(\mathrm{~d}, J=8.7 \mathrm{~Hz}, 2 \mathrm{H}), 5.68(\mathrm{~d}, J=2.4$ $\mathrm{Hz}, 1 \mathrm{H}), 4.00(\mathrm{~s}, 1 \mathrm{H}) ;{ }^{13} \mathrm{C} \mathrm{NMR}\left(75 \mathrm{MHz}, \mathrm{CD}_{3} \mathrm{OD}\right) \delta 178.1$, $174.9,161.2,159.0,140.2,134.0,132.6,128.0,126.8$, 122.2. 116.8, 116.6, 84.0, 57.1; HRMS (FAB) $m / z 327.0857$ $\left[(\mathrm{M}+\mathrm{H})^{+}\right.$, calcd for $\left.\mathrm{C}_{18} \mathrm{H}_{15} \mathrm{O}_{6} 327.0869\right]$.

Compound 3d: 'H NMR (400 MHz, CD $30 D) \delta 7.55(\mathrm{~s}$, $1 \mathrm{H}), 7.36(\mathrm{~s}, 1 \mathrm{H}), 7.15(\mathrm{~d}, J=8.0 \mathrm{~Hz}, 1 \mathrm{H}), 6.86(\mathrm{~s}, 1 \mathrm{H}), 6.81$ $(\mathrm{d}, J=8.0 \mathrm{~Hz}, 1 \mathrm{H}), 6.75(\mathrm{bs}, 2 \mathrm{H}), 5.69(\mathrm{~d}, J=2.0 \mathrm{~Hz}, 1 \mathrm{H})$, 3.98 (bs, $1 \mathrm{H}), 3.87(\mathrm{~s}, 3 \mathrm{H}), 3.78(\mathrm{~s}, 3 \mathrm{H}) ;{ }^{13} \mathrm{C}$ NMR $(100$ $\left.\mathrm{MHz}, \mathrm{CD}_{3} \mathrm{OD}\right) \delta 178.8,175.2,150.4,149.2,149.1,148.0$, $140.2,133.5,127.5,126.8,123.1,119.3,116.5,116.4,114.6$, $110.3,84.4,58.1,56.7,56.4$; HRMS (FAB) $m / z 387.1082$ $\left[(\mathrm{M}+\mathrm{H})^{+}\right.$, calcd for $\left.\mathrm{C}_{20} \mathrm{H}_{19} \mathrm{O}_{8} 387.1080\right]$.

Compound 3f: ${ }^{1} \mathrm{H}$ NMR $\left(300 \mathrm{MHz}, \mathrm{CDCl}_{3}\right) \delta 7.83(\mathrm{~s}$, $1 \mathrm{H}), 7.50-7.28(\mathrm{~m}, 10 \mathrm{H}), 5.81(\mathrm{~s}, 1 \mathrm{H}), 4.25(\mathrm{~s}, 1 \mathrm{H}) ;{ }^{13} \mathrm{C}$ NMR $\left(75 \mathrm{MHz}, \mathrm{CD}_{3} \mathrm{OD}\right) \delta 177.1,174.6,142.3,139.4$, $135.6,131.7,131.1,129.9,129.8,129.4,127.0,126.3,84.5$, 
58.7; HRMS (FAB) $m / 2295.0974\left[(\mathrm{M}+\mathrm{H})^{+}\right.$, caled for $\left.\mathrm{C}_{18} \mathrm{H}_{15} \mathrm{O}_{4} 295.0970\right]$.

Chitin synthase II assay: The strain used in this study is a Sacharomyces cerevisiae ECY38-38A (MATa chsl-23 chs 2::LEU2 call/csd2 tra3-52 trp1-1 leu2-2 pAS6), which can only overexpress the chitin synthase II, that was grown in YPG (yeast extract $1.0 \%$, peptone $2.0 \%$, galactose $2.0 \%$ ). The cells suspended in $50 \mathrm{mM}$ Tris- $\mathrm{HCl}(\mathrm{pH} 7.5)$ containing $5 \mathrm{mM}$ magnesium acetate were broken by vortex mixing with glass beads." And the cell walls were sedimented at $3,000 \times \mathrm{g}$ for $5 \mathrm{~min}$ and supematant fluid was centrifuged at $130,000 \times \mathrm{g}$ for $45 \mathrm{~min}$. The membrane pellet was resuspended in the $50 \mathrm{mM}$ Tris- $\mathrm{HCl}(\mathrm{pH} 7.5)$ containing $33 \%$ glycerol used in the breakage, to a final volume of 1.6 $\mathrm{mL} / \mathrm{g}$ (wet weight) of cells used. The activity of chitin synthase II was measured by the described procedure. ${ }^{9}$ For the proteolytic activation step, reaction mixtures contained $32 \mathrm{mM}$ Tris- $\mathrm{HCl}(\mathrm{pH} 8.0), 1.6 \mathrm{mM}$ cobalt acetate, $1.0 \mathrm{mM}$ UDP- $\left[{ }^{1+} \mathrm{C}\right]-\mathrm{GlcNAc}(400,000 \mathrm{cpm} / \mathrm{mmol}, \mathrm{NEN}), 2 \mathrm{~mL}$ of trypsin at the optimal concentration for activation $(2.0 \mathrm{mg} /$ $\mathrm{mL}$ ), $20 \mu \mathrm{L}$ of membrane suspension, and $14 \mu \mathrm{L}$ of samples in a total volume of $46 \mu \mathrm{L}$. The mixtures were preincubated for $15 \mathrm{~min}$ at $30^{\circ} \mathrm{C}$. Proteolysis was stopped by adding $2 \mu \mathrm{L}$ of a soybean trypsin inhibitor $(4.0 \mathrm{mg} / \mathrm{mL})$ at a concentration 2 times that of trypsin used, and mixtures were placed on ice for $10 \mathrm{~min}$. GlcNAc was added to a final concentration of $32 \mathrm{mM}$ and incubation at $30^{\circ} \mathrm{C}$ was carried out for $90 \mathrm{~min}$. The insoluble chitin formed was assayed by measurement of radioactivity after addition of $10 \%$ trichloroacetic acid and filtration through glass fiber filter $(\mathrm{GF} / \mathrm{C}$,
Whatman). The concentration of protein was measured by the method of Lowry. ${ }^{10}$ Blank values were measured with addition of $25 \%$ aqueous $\mathrm{MeOH}$ instead of both enzyme and sample. Percent inhibition of chitin synthase II activity was calculated by substracting the blank values from both control and test sample values using the following equation: Inhibition $(\%)=[1-$ Sample $(\mathrm{cpm})-\mathrm{Blank}(\mathrm{cpm}) /$ Control (cpm)-Blank (cpm)] $\times 100$.

\section{References}

I. Gooday, G. W. J. Gen. Microbiol. 1977, 99, 1-11.

2. Shaw, J. A.; Mol, P. C.; Bowers, B.; Silverman, S. J.; Valdivieso, M. H.; Duran, A.; Cabib, E. J. Cell Biol, 1991, 114, 1 Il-123.

3. (a) Sburlati, A.; Cabib, E. J. Biol, Chem. 1986, 261, 15147-15152; (b) Silverman, S. J.; Sburlati, A.; Slater, M. L.; Cabib, E. Proc. Natl. Acad. Sci. USA 1988, 85, 4735-4739.

4. (a) Choi, W. J.; Sburlati, A.; Cabib, E. Proc. Natl. Acad. Sci. USA 1994, 91, 4727-4730. (b) Bulawa, C. E. Mol. Cell Biol. 1992, 12. $1764-1776$.

5. Cabib, E.; Sburlati, A.; Bowers, B.; Silverman, \$. J. J. Cell Biol. $1989,108,1665-1672$.

6. Hwang. E.-I.; Yun, B.-S.; Kim, Y.-K.; Kwon, B.-M.; Kim, H.-G.; Lee, H.-B.; Jeong, W.-J.; Kim, S.-U. J. Antibiot. 2000, 53 , $903-$ 911 .

7. Kim, E.; Lee, H. K.; Hwang, E.-I.; Kim, S.-U.; Lee, W. S.; Lee, S.; Jung, S.-H. Synth. Commmin. 2005, 35, 1231-1238.

8. (a) Cartwright, N. J.; Haworth, R. D. J. Chem. Soc, 1944, 535537. (b) Ahmed. R.; Lehrer. M.; Stevenson. R. Tetrahedron 1973. 29, 3753-3759. (c) Pelter, A.; Ward. R. S.; Watson, D. J.; Collins, P.; Kay, I. T. Tetrahedron Left. 1979, 2275-2278.

9. Choi, W. J.; Cabib, E. Anal. Biochem. 1994, 219, 368-372.

10. Lowry, O. H.; Rosebrough, A. L.; Fart, A. L.; Randall, R. J. $J$. Biol. Chem, 1951, 193, 265-275. 